\title{
On bijections between monotone rooted trees and the comb basis
}

\author{
$\mathrm{Fu} \mathrm{Liu} \|^{\dagger}$ \\ Department of Mathematics, University of California, Davis, USA.
}

\begin{abstract}
Let $A$ be an $n$-element set. Let $\mathscr{L} i e_{2}(A)$ be the multilinear part of the free Lie algebra on $A$ with a pair of compatible Lie brackets, and $\mathscr{L} i e_{2}(A, i)$ the subspace of $\mathscr{L} i e_{2}(A)$ generated by all the monomials in $\mathscr{L} i e_{2}(A)$ with $i$ brackets of one type. The author and Dotsenko-Khoroshkin show that the dimension of $\mathscr{L}_{i e_{2}}(A, i)$ is the size of $\mathcal{R}_{A, i}$, the set of rooted trees on $A$ with $i$ decreasing edges. There are three families of bases known for $\mathscr{L} i_{2}(A, i):$ the comb basis, the Lyndon basis, and the Liu-Lyndon basis.

Recently, González D'León and Wachs, in their study of (co)homology of the poset of weighted partitions (which has close connection to $\mathscr{L} i_{2}(A, i)$ ), asked whether there are nice bijections between $\mathcal{R}_{A, i}$ and the comb basis or the Lyndon basis. We give a natural definition for "nice bijections", and conjecture that there is a unique nice bijection between $\mathcal{R}_{A, i}$ and the comb basis. We show the conjecture is true for the extreme cases where $i=0, n-1$.

Résumé. Soit $A$ un ensemble à $n$ éléments. Soit $\mathscr{L} i e_{2}(A)$ la partie multilinéaire de l'algèbre de Lie libre sur $A$ avec une paire de crochets de Lie compatibles et $\mathscr{L} i e_{2}(A, i)$ le sous-espace de $\mathscr{L} i e_{2}(A)$ généré par tous les monômes en $\mathscr{L} i e_{2}(A)$ avec $i$ supports d'un même type. L'auteur et Dotsenko-Khoroshkin montrent que la dimension de $\mathscr{L}_{i e_{2}}(A, i)$ est la taille de la $\mathcal{R}_{A, i}$, l'ensemble des arbres enracinés sur $A$ avec $i$ arêtes décroissantes. Il y a trois familles de bases connues pour $\mathscr{L} i e_{2}(A, i)$ : la base de peigne, la base Lyndon, et la base Liu-Lyndon.

Récemment, Gonzalez, D’ Léon et Wachs, dans leur étude de (co)-homologie de la poset des partitions pondérés, ont demandé si il y a des bijections jolies entre $\mathcal{R}_{A, i}$, et la base de peigne ou la base Lyndon. Nous donnons une définition naturelle de "bijection jolie", et un conjecture qu'il y a une seule bijection jolie entre $\mathcal{R}_{A, i}$, et la base de peigne. Nous montrons que la conjecture est vraie pour les cas extrêmes: $i=0$, et $n-1$.
\end{abstract}

Keywords: free Lie algebra, bijection, rooted trees, comb bases

\section{Introduction}

\subsection{Background}

Let $A=\left\{a_{1}<a_{2}<\cdots<a_{n}\right\}$ be an ordered set and $\mathbf{k}$ a field. Let $\mathscr{L} i e(n)$ or $\mathscr{L} i e(A)$ be the multilinear component of the free Lie algebra on $A$ over k. It is well known that the dimension of $\mathscr{L} i e(n)$ is $(n-1)$ !. Feigin considered the free Lie algebra on $A$ equipped with two compatible Lie brackets $[\cdot, \cdot]$ and $\langle\cdot, \cdot\rangle$, and

${ }^{\dagger} \mathrm{Fu}$ Liu is partially supported by NSF grant DMS-1265702.

1365-8050 @ 2015 Discrete Mathematics and Theoretical Computer Science (DMTCS), Nancy, France 
its multilinear component which we denote by $\mathscr{L} i e_{2}(n)$ or $\mathscr{L} i e_{2}(A)$. Further, he conjectured the dimension of $\mathscr{L} i e_{2}(n)$ to be $n^{n-1}$. His conjecture was proved by the author [7] and Dotsenko-Khoroshkin [2, 3]. In fact, a refinement of Feigin's conjecture was obtained in [7] as well: let $\mathscr{L} i e_{2}(n, i)$ (or $\mathscr{L} i e_{2}(A, i)$ ) be the subspace of $\mathscr{L} i e_{2}(n)$ (or $\mathscr{L} i e_{2}(A)$ ) generated by all the monomials in $\mathscr{L} i e_{2}(n)$ (or $\mathscr{L} i e_{2}(A)$ ) with exactly $i[\cdot, \cdot]$ 's (and $n-1-i\langle\cdot, \cdot\rangle$ 's), then

$$
\operatorname{dim} \mathscr{L}_{i e_{2}}(A, i)=\left|\mathcal{R}_{A, i}\right|,
$$

where $\mathcal{R}_{A, i}$ is the set of rooted trees on $A$ with $i$ decreasing edges. In [2, 3], Dotsenko and Khoroshkin use the theory of operads to prove Feigin's conjecture. They also obtain character formulas for the representation of the symmetric groups $\mathfrak{S}_{n}$ and the $S L_{2}$ group in $\mathscr{L} i e_{2}(n)$. Although $(1.1)$ is not derived directly in their work, it follows from their character formulas and a result of Drake [4].

Another interesting property of $\mathscr{L} i e(n)$ is its close connection to the (co)homology of the partition lattice $\Pi_{n}$. The symmetric group $\mathfrak{S}_{n}$ acts naturally on $\mathscr{L} i e(n)$, so we can consider $\mathscr{L} i e(n)$ an $\mathfrak{S}_{n}$-module. At the same time, $\mathfrak{S}_{n}$ also acts naturally on $\Pi_{n}$ and this action induces isomorphic representations of $\mathfrak{S}_{n}$ on the unique nonvanishing reduced simplicial homology $\tilde{H}_{n-3}\left(\bar{\Pi}_{n}\right)$ and cohomology $\tilde{H}^{n-3}\left(\bar{\Pi}_{n}\right)$ of the order complex $\Delta\left(\bar{\Pi}_{n}\right)$ of the proper part $\bar{\Pi}_{n}$ of $\Pi_{n}$. It is a classical result that

$$
\tilde{H}_{n-3}\left(\bar{\Pi}_{n}\right) \cong \mathfrak{S}_{n} \mathscr{L} i e(n) \otimes \operatorname{sgn}_{n},
$$

where $\operatorname{sgn}_{n}$ is the sign representation of $\mathfrak{S}_{n}$.

Dotsenko and Khoroshkin [2] introduced a weighted version of $\Pi_{n}$, denoted by $\Pi_{n}^{w}$ and called the poset of weighted partitions. (The precise defintion of $\Pi_{n}^{w}$ is not important to us in this paper.) The weighted partition poset has one unique minimal element, denoted by $\hat{0}$, and $n$ maximal elements, denoted by $[n]^{i}$ for $i=0,1, \ldots, n-1$. The (co)homology of maximal open intervals $\left(0,[n]^{i}\right)$ is studied. Vallette $[8]$ and Dotsenko-Khoroshkin [3] show, using the theory of operads, that

$$
\tilde{H}_{n-3}\left(\left(\hat{0},[n]^{i}\right)\right) \simeq_{\mathfrak{S}_{n}} \mathscr{L} i e_{2}(n, i) \otimes \operatorname{sgn}_{n} .
$$

Since $\left(0,[n]^{i}\right) \cong \bar{\Pi}_{n}$ and $\mathscr{L} i e_{2}(n, i) \cong \mathscr{L} i e(n)$ when $i=0$ or $n-1$, their result generalizes $(1.2)$.

In [6], González D'león and Wachs extend various classical results of $\Pi_{n}$ to $\Pi_{n}^{w}$. In particular, they establish a strong connection between cohomology of open intervals $\left(\hat{0},[n]^{i}\right)$ and $2 v$-colored binary trees, which give another proof for $(1.3)$ and provide a way to construct bases for $\tilde{H}^{n-3}\left(\left(\hat{0},[n]^{i}\right)\right)$ from bases for $\mathscr{L}_{i e_{2}}(n, i)$. They also construct a basis for $\tilde{H}_{n-3}\left(\left(\hat{0},[n]^{i}\right)\right)$ from $\mathcal{R}_{A, i}$.

\subsection{Questions and results}

There are three families of bases known for $\mathscr{L} i e_{2}(A, i)$ (and thus for $\tilde{H}^{n-3}\left(\left(\hat{0},[n]^{i}\right)\right)$ ) summarized in [6] by González D'León and Wachs in their study of (co)homology of weighted partition poset. We list them here, following the notations in [6]:

- $\mathrm{Comb}_{A, i}^{2}$ : Bershtein-Dotsenk-Khoroshkin [1] introduced a comb basis, for $\mathscr{L} i e_{2}(A, i)$ generalizing Wachs' comb basis [9] for $\mathscr{L} i e(A)$.

- $\operatorname{Liu}_{A, i}^{2}$ : The author [7] introduced a Liu-Lyndon basis for $\mathscr{L} i e_{2}(A, i)$ generalizing the standard Lyndon basis for $\mathscr{L} i e(A)$. 
- $\operatorname{Lyn}_{A, i}^{2}$ : González D'león-Wachs [6] constructed another basis for $\mathscr{L} i e_{2}(A, i)$ that generalizes the standard Lyndon basis. We will refer to it as the Lyndon basis.

Since the dimension of $\mathscr{L} i e_{2}(A, i)$ is known to be the size of $\mathcal{R}_{A, i}$, we have

$$
\left|\mathcal{R}_{A, i}\right|=\left|\operatorname{Comb}_{A, i}^{2}\right|=\left|\operatorname{Liu}_{A, i}^{2}\right|=\left|\operatorname{Lyn}_{A, i}^{2}\right|
$$

It is thus natural to ask whether one can give bijections between the above four sets. In [7], the author gives a bijection between $\mathcal{R}_{A, i}$ and $\operatorname{Liu}_{A, i}$. In [5], González D'león constructs a bijection between $\operatorname{Comb}_{A, i}^{2}$ and $\operatorname{Lyn}_{n, i}^{2}$. Given these results, González D'león and Wachs ask whether there are nice bijections between $\mathcal{R}_{A, i}$ and $\operatorname{Comb}_{A, i}^{2}$ or $\operatorname{Lyn}_{A, i}^{2}$ [6, Remark 5.11]. The motivation of this paper is to answer their question.

Before we can answer the question of González D’león and Wachs, we need to clarify what bijections are "nice". We give such a definition in Section 3, where good-pair bijections are defined. With this definition, we conjecture the following:

Conjecture 1.1 There exists a unique good-pair bijection from $\mathcal{R}_{A, i} \rightarrow \operatorname{Comb}_{A, i}^{2}$ for any nonempty finite ordered set $A$ and $0 \leq i \leq|A|-1$.

Below is the main result of the paper.

Theorem 1.2 Conjecture 1.1 holds for $i=0$ and $i=n-1$.

We finish this part with a potential consequence of answering González D’león and Wachs' question. In [5], González D'león generalizes $\mathscr{L} i e_{2}(n)$ to $\mathscr{L} i e_{k}(n)$, the multilinear part of the free Lie algebra with $k$ compatible brackets, and introduces a new corresponding poset of weighted partition $\Pi_{n}^{k}$ that generalizes $\Pi_{n}^{w}$. He obtains various dimension formulas for $\mathscr{L} i e_{k}(n)$ and generalizations of the comb basis $\operatorname{Comb}_{A, i}^{2}$ and the Lyndon basis $\operatorname{Lyn}_{A, i}^{2}$. Generalizing the techniques in [6], he shows these bases also provide bases for cohomology of maximal open intervals of $\Pi_{n}^{k}$. However, bases for homology of $\Pi_{n}^{k}$ are still waiting to be found. One key ingredient used in [6] to find a basis for $\tilde{H}_{n-3}\left(\left(\hat{0},[n]^{i}\right)\right)$ is the bijection from $\mathcal{R}_{A, i}$ to $\mathrm{Liu}_{A, i}^{2}$ given in [7]. Since neither bases for $\mathscr{L} i e_{k}(n)$ constructed in [5] is a generalization of $\mathrm{Liu}_{A, i}^{2}$, it is hard to generalize the method used for $\Pi_{n}^{w}$ to $\Pi_{n}^{k}$. However, if we are able to find a nice bijection from $\mathcal{R}_{A, i}$ to either $\operatorname{Comb}_{A, i}^{2}$ or $\operatorname{Lyn}_{A, i}^{2}$, we can hope to generalize it and find a basis for the homology of maximal open intervals of $\Pi_{n}^{k}$.

\section{Rooted trees and the comb basis}

In this section, we will introduce the main combinatorial objects that are going to be used in this paper.

Definition 2.1 A tree is a connected acyclic graph. A rooted tree is a tree with one special vertex, which we call it the root of the tree. Let $\mathcal{R}_{A}$ be the set of all rooted trees on the n-element set $A$, i.e., all the rooted trees with $n$ vertices that are labeled by the elements in $A$.

For any edge $\{i, j\}$ in a rooted tree, if $i$ is closer to the root than $j$, we call $i$ the parent of $j$ and $j$ a child of $i$. Furthermore, if $i$ is the parent of $j$, we say $\{i, j\}$ an increasing edge if $i<j$, and a decreasing edge if $i>j$. For convenience, we color each increasing edge blue and each decreasing edge red.

Let $\mathcal{R}_{A, i}$ be the set of rooted trees on $A$ with $i$ decreasing/blue edges (and $n-1-i$ increasing/blue edges). 
Note that for any rooted tree in $\mathcal{R}_{A, 0}$, all of its edge are increasing. Thus, we call trees in $\mathcal{R}_{A, 0}$ increasing trees. Similarly, we call trees in $\mathcal{R}_{A, n-1}$ decreasing trees.

A monotone tree is either an increasing tree or a decreasing tree.

See Figure 1 for the nine rooted trees in $\mathcal{R}_{A}$ with $n=3$, where the first two trees on the first row are increasing trees in $\mathcal{R}_{A, 0}$, the last two trees on the first row are decreasing trees in $\mathcal{R}_{A, 2}$, and the five trees on the second row are in $\mathcal{R}_{A, 1}$.
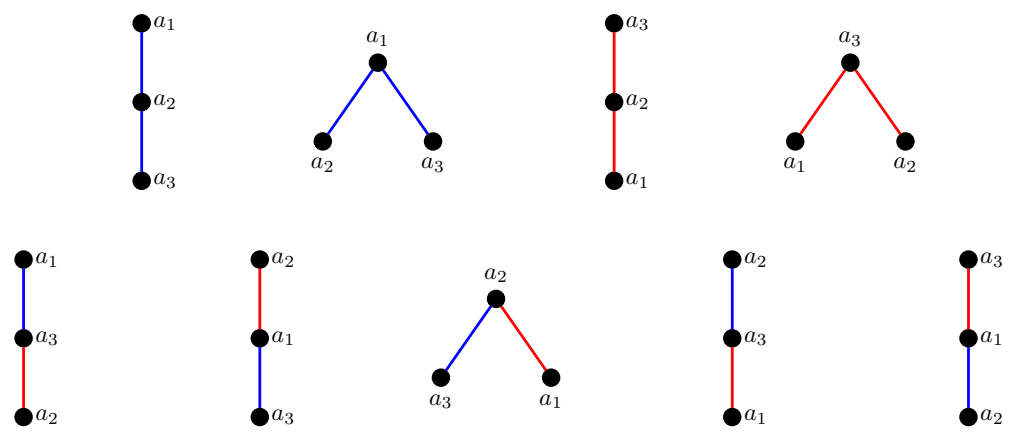

Fig. 1: Rooted trees in $\mathcal{R}_{A}$ with $n=3$

A vertex in a rooted tree is a leaf if it does not have any children, and is an internal vertex otherwise.

Definition 2.2 A binary tree is an ordered (rooted) tree, where all of its internal vertices have exactly two children.

A $2 v$-colored binary tree is a binary tree whose internal vertices are colored by red or blue. We denote by $\mathcal{B T}_{A}$ the set of all $2 v$-colored binary trees whose leaves are labeled by $A$, and by $\mathcal{B T}_{A, i}$ the set of all $2 v$-colored binary trees whose leaves are labeled by $A$ and $i$ of whose internal vertices are red.

See Figure 2 for an example of $2 v$-colored binary tree.

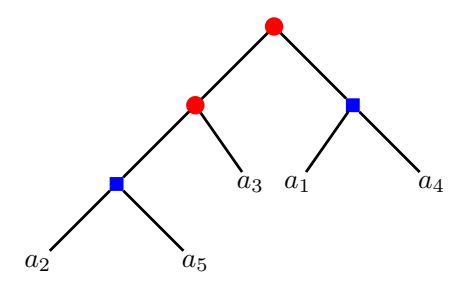

Fig. 2: Example of a tree in $\mathcal{B} \mathcal{T}_{A, 2}$ with $n=5$

A labeled binary tree is normalized if the leftmost leaf of each subtree has the smallest label in the subtree.

Definition 2.3 A tree in $\mathcal{B T}_{A}$ is a $2 \mathrm{v}$-colored comb if it is normalized and satisfies the following coloring restriction: for each internal vertex $i$ whose right child $j$ is not a leaf, $i$ is colored red and $j$ is colored blue. 
We denote by $\mathrm{Comb}_{A}^{2}$ the set of all $2 v$-colored combs in $\mathcal{B T}_{A}$, and by $\mathrm{Comb}_{A, i}^{2}$ the set of all $2 v$-colored combs in $\mathcal{B T}_{A, i}$.

See Figure 3 for all the nine combs in $\mathrm{Comb}_{A}^{2}$ with $n=3$, where the first two trees on the first row are in $\mathrm{Comb}_{A, 0}^{2}$, the last two trees on the first row are in $\mathrm{Comb}_{A, 2}^{2}$, and the five trees on the second row are in $\mathrm{Comb}_{A, 1}^{2}$.

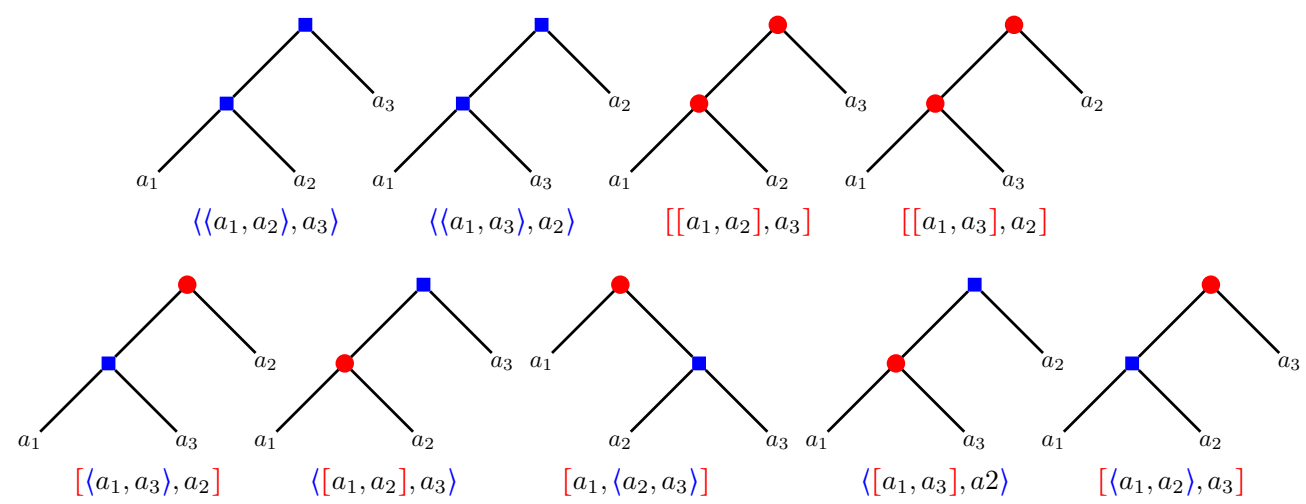

Fig. 3: $2 v$-colored combs in $\operatorname{Comb}_{A}^{2}$ with $n=3$

There is a canonical bijection between $\mathcal{B} \mathcal{T}_{A, i}$ and all the monomials in $\mathscr{L}_{i e_{2}}(A, i)$ : given a $2 v$-colored binary tree in $\mathcal{B} \mathcal{T}_{A, i}$, each leaf denotes a letter in $A$, and we can construct a monomial in $\mathscr{L} i e_{2}(A, i)$ recursively by interpreting each internal vertex as a bracket of the left and right subtrees, with red vertices corresponding to $[\cdot, \cdot]$ and blue vertices corresponding to $\langle\cdot, \cdot\rangle$. For example, in Figure 3, each comb corresponds to the monomial shown below it. Therefore, we can consider that $\mathscr{L} i e_{2}(A, i)$ is spanned by elements of $\mathcal{B T}_{A, i}$ and use $\mathcal{B} \mathcal{T}_{A, i}$ to describe bases of $\mathscr{L} i e_{2}(A)$.

Bershtein, Dotsenko and Khoroshkin show in [1] that $\mathrm{Comb}_{A, i}^{2}$, considered as a set of monomials, is a basis for $\mathscr{L} i e_{2}(A, i)$, and we call it the comb basis.

From now on, we will always consider $\mathcal{B T}_{A}$ and $\operatorname{Comb}_{A}^{2}$ as sets of monomials of $\mathscr{L}_{i e_{2}}(A)$. (We only introduce the binary tree terminology because it is easier to be used to describe the combs.)

\section{Good-pair bijections}

In this section, we will define good-pair bijections, which we consider to be "nice" bijections, and reduce Conjecture 1.1 to a new conjecture (Conjecture 3.9). We will also explain why our definition of "good-pair bijection" is natural.

Definition 3.1 We say $m \in \mathcal{B T}_{A}$ and $G \in \mathcal{R}_{A}$ form a good pair if one of the following is satisfied:

(i) $|A|=1$.

(ii) Suppose $|A| \geq 2$ and $m=\left\{m_{1}, m_{2}\right\}$, where $\{\cdot, \cdot\}=[\cdot, \cdot]$ or $\langle\cdot, \cdot\rangle$. There exists an edge e of $G$ such that: 
1. e has the same color as $\{\cdot, \cdot\}$, i.e., e is red if $\{\cdot, \cdot\}=[\cdot, \cdot]$ and $e$ is blue if $\{\cdot, \cdot\}=\langle\cdot, \cdot\rangle$;

2. By removing e from $G$, we obtain two rooted trees $G_{1}$ and $G_{2}$ such that $m_{i}$ and $G_{i}$ form a good pair for each $i$.

It follows immediately from the definition that if $m \in \mathcal{B} \mathcal{T}_{A}$ and $G \in \mathcal{R}_{A}$ form a good pair, then $m \in \mathcal{B T}_{A, i}$ and $G \in \mathcal{R}_{A, i}$ for some $i$.

Example 3.2 Below we give all the good pairs between elements in $\mathcal{R}_{A}$ and $\mathcal{B T}_{A}$ when $|A|=2$.

The rooted tree $\left.\right|_{a_{1}} ^{a_{2}} \quad$ forms a good pair with $\left\langle a_{1}, a_{2}\right\rangle$ and $\left\langle a_{2}, a_{1}\right\rangle$.

Example 3.3 In Figure 4, $G_{1}$ and $G_{2}$ are the (only) two rooted trees in $\mathcal{R}_{A, 0}$, and $m_{1}$ and $m_{2}$ are two monomials in $\mathcal{B T}_{A, 0}$. We summarize the good-pair relations between $\left\{m_{1}, m_{2}\right\}$ and $\left\{G_{1}, G_{2}\right\}$ below.
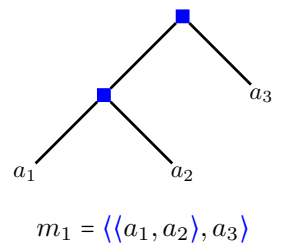

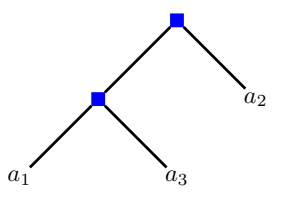

$m_{2}=\left\langle\left\langle a_{1}, a_{3}\right\rangle, a_{2}\right\rangle$

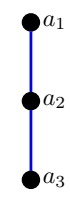

$G_{1}$

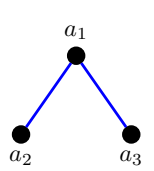

$G_{2}$

Fig. 4:

- $G_{1}$ forms a good pair with $m_{1}$, but not with $m_{2}$. (One sees that in order to be a good pair with $m_{2}$, a rooted tree has to have a blue edge connecting $a_{1}$ and $a_{3}$. However, $G_{1}$ does not have such an edge.)

- $G_{2}$ forms a good pair with both $m_{1}$ and $m_{2}$.

Definition 3.4 Given $B \subseteq \mathcal{B} \mathcal{T}_{A, i}$ a basis for $\mathscr{L} i_{2}(A, i)$, we say a bijection $\psi: \mathcal{R}_{A, i} \rightarrow B$ is a good-pair bijection if $\psi(G)$ and $G$ form a good pair for each $G \in \mathcal{R}_{A, i}$.

Example 3.5 Suppose $n=3$. Let $m_{1}, m_{2}, G_{1}$ and $G_{2}$ be as in Figure 4 . Note that $\left\{m_{1}, m_{2}\right\}=\mathrm{Comb}_{A, 0}^{2}$ is the comb basis for $\mathscr{L} i e_{2}(A, i)$.

Clearly, the map that maps $G_{i}$ to $m_{i}$ for $i=1,2$ is a good-pair bijection. Furthermore, we observe that it is the unique good-pair bijection from $\mathcal{R}_{A, 0}$ to $B$.

Because of the observation in the above example, we modify question of González D’León and Wachs:

Question 3.6 For each of the three known bases $\operatorname{Comb}_{A, i}^{2}$, $\operatorname{Liu}_{A, i}^{2}$, and $\operatorname{Lyn}_{A, i}^{2}$, does good-pair bijection exist? If so, is it unique?

The following lemma gives one way to prove both the existence and uniqueness of a good-pair bijection. 
Lemma 3.7 Let $B \subseteq \mathcal{B T}_{A, i}$ be a basis for $\mathscr{L}_{i e_{2}}(A, i)$. There exist a total ordering on $\mathcal{R}_{A, i}: G_{1}, G_{2}, \ldots$ and a total ordering on $B: b_{1}, b_{2}, \ldots$ such that

(i) $b_{i}$ and $G_{i}$ form a good pair for each $i$;

(ii) $b_{i}$ and $G_{j}$ does not form a good pair unless $i \leq j$.

Then there is a unique good-pair bijection from $\mathcal{R}_{A, i}$ to $B$.

Proof: First, mapping $G_{i}$ to $b_{i}$ for each $i$ gives a good-pair bijection. On the other hand, given a good-pair bijection $\psi$, since $G_{1}$ is good pair with nothing other than $b_{1}, \psi$ has to map $G_{1}$ to $b_{1}$. As a result, it is forced to map $G_{2}$ to $b_{2}$, and so on. Hence, the good-pair bijection is unique.

In fact, in [7], the author shows that the hypothesis of Lemma 3.7]is satisfied for the Liu-Lyndon basis $\operatorname{Liu}_{A, i}^{2}$. Hence, we immediately have the following fact:

Fact 3.8 There exists a unique good-pair bijection from $\mathcal{R}_{A, i}$ to $\operatorname{Liu}_{A, i}^{2}$ and it is the one constructed in [7].

Therefore, Question 3.6 is only open for the comb basis $\mathrm{Comb}_{A, i}^{2}$ and the Lyndon basis $\operatorname{Lyn}_{A, i}^{2}$. After checking small examples of comb bases, we conjecture that the hypothesis of Lemma 3.7 holds for the comb basis.

Conjecture 3.9 There exist a total ordering on $\mathcal{R}_{A, i}: G_{1}, G_{2}, \ldots$ and a total ordering on $\mathrm{Comb}_{A, i}^{2}$ : $m_{1}, m_{2}, \ldots$ such that

(i) $m_{i}$ and $G_{i}$ form a good pair for each $i$;

(ii) $m_{i}$ and $G_{j}$ does not form a good pair unless $i \leq j$.

By Lemma 3.7. Conjecture 3.9 implies Conjecture 1.1

Example 3.10 We've verified that Conjecture 3.9 is true for $n \leq 4$. Hence, Conjecture 1.1 holds for $n \leq 3$. The situations for $n=1$ and 2 are trivial. When $n=3$, we order $\mathcal{R}_{A, i}$ and $\operatorname{Comb}_{A, i}$ as they are shown in Figures 1 and 3 One checks that the orderings satisfying conditions (i) and (ii) of Conjecture 3.9

There are $4^{3}=64$ rooted trees and combs when $n=4$. So we omit the details here.

Remark 3.11 For the Lyndon basis $\operatorname{Lyn}_{A, i}^{2}$, we've checked that the hypothesis of Lemma 3.7 holds for $n$ up to 3. However, we have not had chance to check the case for $n=4$. Since for $n \leq 3$, the size of each basis is very small, we do not think we have enough data to make similar conjectures.

\section{Why good-pair?}

We finish this section with an explanation of why we consider good-pair bijections are nice bijections. In fact, the definition of "good pair" has close connection to both work in [7] and [6].

In [7], the author constructs a monomial $b_{G}$ from each $G \in \mathcal{R}_{A, i}$ and shows that $\operatorname{Liu}_{A, i}^{2}:=\left\{b_{G} \mid G \epsilon\right.$ $\left.\mathcal{R}_{A, i}\right\}$ is a basis for $\mathscr{L} i e_{2}(A, i)$. One main technique used in the proof is that a complementary space $\mathscr{E} i l_{2}(A)$ is introduced where rooted trees are elements of $\mathscr{E} i l_{2}(A)$, and a pairing between $\mathscr{L} i e_{2}(A)$ and $\mathscr{E} i l_{2}(A)$ are defined and shown to be perfect. Then the pairing of $b_{G}$ and $G^{\prime}$ is nonzero if and only if $b_{G}$ and $G^{\prime}$ form a good pair, for any $G, G^{\prime} \in \mathcal{R}_{A, i}$. 
In [6], González D'León and Wachs associate $\bar{c}_{T}$, a maximal chain in $\left(\hat{0},[n]^{i}\right)$, to each $T \in \mathcal{B T}_{A, i}$. This association provides a connection between bases for $\mathscr{L} i e_{2}(A, i)$ and bases for $\tilde{H}^{n-3}\left(\left(\hat{0},[n]^{i}\right)\right)$. More precisely, for any basis $B \subseteq \mathcal{B} \mathcal{T}_{A, i}$ of $\mathscr{L} i e_{2}(A, i),\left\{\bar{c}_{T} \mid T \in B\right\}$ is a basis for $\tilde{H}^{n-3}\left(\left(\hat{0},[n]^{i}\right)\right)$. They also associate $\rho_{G}$, a fundamental cycle of the spherical complex $\Delta\left(\bar{\Pi}_{G}\right)$, to each $G \in \mathcal{R}_{A, i}$, and show that $\left\{\rho_{G} \mid G \in \mathcal{R}_{A, i}\right\}$ is a basis for $\tilde{H}_{n-3}\left(\left(\hat{0},[n]^{i}\right)\right)$. Also, when the (co)homology is defined in [6], the chain and cochain spaces have been identified using the natural bases. This identification is given by the bilinear form $\langle\langle\cdot, \cdot\rangle\rangle$. Then we have that $\left\langle\left\langle\rho_{G}, \bar{c}_{T}\right\rangle\right\rangle \neq 0$ if and only if $T$ and $G$ form a good pair.

Therefore, one sees that "good-pair" really is a natural way to define a "nice" bijection from $\mathcal{R}_{A, i}$ to a basis of $\mathscr{L} i e_{2}(A, i)$. In particular, because of its connection to bilinear form defined between homology and cohomology in [6], like we've discussed in the introduction, if one is able to find a good-pair bijection from $\mathcal{R}_{A, i}$ to either $\mathrm{Comb}_{A, i}^{2}$ or $\operatorname{Lyn}_{A, i}^{2}$, it is very likely to be generalized and be used to find a basis for the homology of maximal open intervals of $\pi_{n}^{k}$ introduced by González D’León.

\section{The unique bijection between $\mathcal{R}_{A, 0}$ and $\mathrm{Comb}_{A, 0}^{2}$}

In this section, we will show our conjectures are true for $i=0$. Since all the trees in $\mathcal{R}_{A, 0}$ are increasing trees, we write $\mathcal{R}_{A, 0}$ as $\mathcal{R}_{A}^{i}$.

The main result of this section is the following:

Theorem 4.1 Conjecture 3.9 holds for $i=0$. Hence, Conjecture 1.1 holds for $i=0$.

Observation 4.2 We see that $\mathrm{Comb}_{A, 0}^{2}$ consists of all monomials of the form

$$
\left\langle\left\langle\cdots\left\langle\left\langle a_{1}, a_{i_{2}}\right\rangle, a_{i_{3}}\right\rangle, \cdots, a_{i_{n-1}}\right\rangle, a_{i_{n}}\right\rangle,
$$

where $i_{2} i_{3} \cdots i_{n-1} i_{n}$ is a permutation on $\{2,3, \ldots, n\}$.

\subsection{A good-pair bijection}

We will first construct a bijection from $\mathcal{R}_{A}^{i}$ to $\operatorname{Comb}_{A, 0}^{2}$ and show it is a good-pair bijection. We need a preliminary definition.

Definition 4.3 Let $|A| \geq 2$. For each $G \in \mathcal{R}_{A}^{i}$, the cutting path, the cutting edge, and the cutting node of $G$, denoted by $\mathrm{CP}(G), \mathrm{CE}(G)$, and $\mathrm{CN}(G)$ are defined as below:

Let $v_{0}:=a_{1}$ be the root and for each $i \geq 0$, if $v_{i}$ is not a leaf, let $v_{i+1}$ be the smallest child of $v_{i}$. Suppose $v_{k}$ is a leaf. Then let

$$
\mathrm{CP}(G):=v_{0}-v_{1}-v_{2}-\cdots-v_{k},
$$

and let $\mathrm{CE}(G):=\left\{v_{k-1}, v_{k}\right\}$ be the last edge in the cutting path, and $\mathrm{CN}(G):=v_{k}$ be the leaf in the path.

Now we are ready to define the desired bijection between $\mathcal{R}_{A}^{i}$ and $\mathrm{Comb}_{A, 0}^{2}$.

Definition 4.4 We define a map $\varphi_{A}: \mathcal{R}_{A}^{i} \rightarrow \mathrm{Comb}_{A, 0}^{2}$ recursively in the following way. Let $G \in \mathcal{R}_{A}^{i}$.

(i) If $A=\{a\}$ has only one element, we define $\varphi_{A}(G):=a$.

(ii) Otherwise, if $|A| \geq 2$, we define

$$
\varphi_{A}(G):=\left\langle\varphi_{A \backslash\{C N(G)\}}(G \backslash \mathrm{CN}(G)), \mathrm{CN}(G)\right\rangle,
$$

where $G \backslash \mathrm{CN}(G)$ is the tree obtained from $G$ by removing the leaf $\mathrm{CN}(G)$ and its adjacent edge $\mathrm{CE}(G)$, so it is in $\mathcal{R}_{A \backslash \mathrm{CN}(G)}^{i}$. 
When there is no confusion of the set $A$, we will omit the subscript A from $\varphi_{A}$ and just write $\varphi$.

It is easy to see that $\varphi(G)$ is always in the form of 4.1. Hence, the map $\varphi$ is well-defined.

Example 4.5 Let $G_{1}$ and $G_{2}$ be the two rooted trees in Figure 4 Then

$$
\begin{array}{rll}
\mathrm{CP}\left(G_{1}\right)=a_{1}-a_{2}-a_{3} & \text { and } & \varphi\left(G_{1}\right)=\left\langle\left\langle a_{1}, a_{2}\right\rangle, a_{3}\right\rangle ; \\
\mathrm{CP}\left(G_{2}\right)=a_{1}-a_{2} & \text { and } & \varphi\left(G_{2}\right)=\left\langle\left\langle a_{1}, a_{3}\right\rangle, a_{2}\right\rangle .
\end{array}
$$

Proposition 4.6 $\varphi$ is a good-pair bijection from $\mathcal{R}_{A}^{i}$ to $\operatorname{Comb}_{A, 0}^{2}$.

Since it is clear that $G$ and $\varphi(G)$ form a good pair for each $G \in \mathcal{R}_{A}^{i}$, in order to prove Proposition 4.6 . we only need to construct an inverse map $\varphi^{-1}$ from $\operatorname{Comb}_{A, 0}^{2}$ to $\mathcal{R}_{A}^{i}$.

Definition 4.7 Suppose $|A| \geq 2$. Let a be an element of $A$ that is not $a_{1}$, and let $A^{\prime}=A \backslash\{a\}$. Suppose $G^{\prime} \in \mathcal{R}_{A^{\prime}}^{i}$, with

$$
\mathrm{CP}\left(G^{\prime}\right)=v_{0}-v_{1}-v_{2}-\cdots-v_{k}
$$

and let

$$
\ell:=\max \left\{i \mid v_{i}<a\right\} .
$$

(So we have $a_{1}=v_{0}<v_{1}<\cdots<v_{\ell}<a<v_{\ell+1}<\cdots<v_{k}$.) We define the i-merge of $G^{\prime}$ and $a$, denoted by $G^{\prime} \triangleleft a$, to be the increasing tree obtained by connecting a to the vertex $v_{\ell}$ of $G^{\prime}$.

Definition 4.8 We define a map $\varphi_{A}^{-1}: \mathrm{Comb}_{A, 0}^{2} \rightarrow \mathcal{R}_{A}^{i}$ in the following way. Let $m \in \mathrm{Comb}_{A, 0}^{2}$.

(i) If $A=\{a\}$ has only one element, we define $\varphi_{A}^{-1}(m):=a$.

(ii) Otherwise, Suppose $|A| \geq 2$ and $m$ is in the form of 4.1. Let

$$
\varphi_{A}^{-1}(m):=\left(\left(\cdots\left(\left(a_{1} \triangleleft a_{i_{2}}\right) \triangleleft a_{i_{3}}\right) \triangleleft \cdots \triangleleft a_{i_{n-1}}\right) \triangleleft a_{i_{n}}\right) .
$$

It is easy to verify that $\varphi_{A}$ and $\varphi_{A}^{-1}$ are inverse to each other. Therefore, we complete the proof of Proposition 4.6 .

\subsection{An ordering on cutting paths}

We will define an ordering on sequences that are made from the ordered set $A$.

Definition 4.9 Suppose $\alpha=\alpha_{1}-\alpha_{2}-\cdots-\alpha_{s}$ and $\beta=\beta_{1}-\beta_{2}-\cdots-\beta_{t}$, where $\alpha_{i}, \beta_{j} \in A$. We write

$$
\alpha<_{\operatorname{mlex}} \beta
$$

if one of the following is satisfied:

a) There exists $1 \leq r \leq \min (s, t)$ such that $\alpha_{i}=\beta_{i}$ for each $i<r$ and $\alpha_{r}<\beta_{r}$.

b) $\beta$ is a proper subsequence of $\alpha$; that is, $t<s$ and $\alpha_{i}=\beta_{i}$ for each $i \leq t$.

We call the above ordering the modified lex(icographical) order. (Note that if we replace condition b) with " $\alpha$ is a proper subsequence of $\beta$ ", we recover the regular lex order.) One can verify $<_{\operatorname{mlex}}$ is a well-defined ordering. 
Example 4.10 Let $G_{1}$ and $G_{2}$ be the two rooted trees in Figure 4 Then

$$
\mathrm{CP}\left(G_{1}\right)=a_{1}-a_{2}-a_{3}<_{\text {mlex }} \operatorname{CP}\left(G_{2}\right)=a_{1}-a_{2} .
$$

We have the following two important lemmas stating that the i-merge operation $\triangleleft$ minimizes the cutting path, and preserving the orderings of cutting paths.

Lemma 4.11 Suppose $|A| \geq 2$. Let a be an element of $A$ that is not $a_{1}$, and let $A^{\prime}=A \backslash\{a\}$. Suppose $G^{\prime} \in \mathcal{R}_{A^{\prime}}^{i}$ and $G \in \mathcal{R}_{A}^{i}$ is obtained by connecting a to a vertex of $G^{\prime}$. Then we have the followings:

a) If $G=G^{\prime} \triangleleft a$, then $\mathrm{CP}(G)<_{\text {mlex }} \mathrm{CP}\left(G^{\prime}\right)$;

b) Otherwise, $\operatorname{CP}(G)=\operatorname{CP}\left(G^{\prime}\right)$.

Hence, we always have $\mathrm{CP}\left(G^{\prime} \triangleleft a\right) \leq_{\operatorname{mlex}} \mathrm{CP}(G)$.

Proof: Suppose $\mathrm{CP}\left(G^{\prime}\right)$ and $\ell$ are given as in Definition 4.7 Then

$$
\mathrm{CP}\left(G^{\prime} \triangleleft a\right)=v_{0}-v_{1}-\cdots-v_{\ell}-a .
$$

Thus, a) follows immediately from the definition of the modified lex order.

Suppose $G \neq\left(G^{\prime} \triangleleft a\right)$. Let $v$ be the vertex in $G^{\prime}$ that $a$ is connected to. Then $v \neq v_{\ell}$. If $v \neq v_{i}$ for any $i$, it is clear that we have $\mathrm{CP}(G)=\mathrm{CP}\left(G^{\prime}\right)$. Suppose $v=v_{i}$ for some $i \neq \ell$. Since $a<v_{i}$ for $i>\ell$ and $\{v, a\}$ is an increasing edge, we must have that $i<\ell$. Then one checks that $\mathrm{CP}(G)=\mathrm{CP}\left(G^{\prime}\right)$.

Lemma 4.12 Suppose $|A| \geq 2$. Let a be an element of $A$ that is not $a_{1}$, and let $A^{\prime}=A \backslash\{a\}$. Suppose $G_{1}, G_{2} \in \mathcal{R}_{A^{\prime}}^{i}$ with $\mathrm{CP}\left(G_{1}\right) \leq_{\text {mlex }} \mathrm{CP}\left(G_{2}\right)$. Then $\mathrm{CP}\left(G_{1} \triangleleft a\right) \leq_{\text {mlex }} \mathrm{CP}\left(G_{2} \triangleleft a\right)$.

Proof: If $\mathrm{CP}\left(G_{1}\right)=\mathrm{CP}\left(G_{2}\right)$, by the definition of i-merge, it's clear that $\mathrm{CP}\left(G_{1} \triangleleft a\right)=\mathrm{CP}\left(G_{2} \triangleleft a\right)$. Suppose

$$
\mathrm{CP}\left(G_{1}\right)=\alpha_{1}-\alpha_{2}-\cdots-\alpha_{s}<_{\text {mlex }} \mathrm{CP}\left(G_{2}\right)=\beta_{1}-\beta_{2}-\cdots-\beta_{t},
$$

and let

$$
\ell:=\max \left\{i \mid \alpha_{i}<a\right\} .
$$

Then one of the conditions in Definition 4.9 is satisfied. Suppose condition b) of Definition 4.9 is satisfied; that is, $\mathrm{CP}\left(G_{2}\right)$ is a proper subsequence of $\mathrm{CP}\left(G_{1}\right)$. Then

$$
\mathrm{CP}\left(G_{1} \triangleleft a\right)<_{\text {mlex }} \mathrm{CP}\left(G_{2} \triangleleft a\right) \text { if } \ell>t, \quad \text { and } \quad \mathrm{CP}\left(G_{1} \triangleleft a\right)=\mathrm{CP}\left(G_{2} \triangleleft a\right) \text { if } \ell \leq t .
$$

Suppose condition a) of Definition 4.9 is satisfied. Then

$$
\mathrm{CP}\left(G_{1} \triangleleft a\right)<_{\text {mlex }} \mathrm{CP}\left(G_{2} \triangleleft a\right) \text { if } \ell \geq r, \quad \text { and } \quad \mathrm{CP}\left(G_{1} \triangleleft a\right)=\mathrm{CP}\left(G_{2} \triangleleft a\right) \text { if } \ell<r .
$$

Corollary 4.13 Suppose $m \in \mathrm{Comb}_{A, 0}^{2}$ and $G \in \mathcal{R}_{A}^{i}$ form a good pair. Then

$$
\mathrm{CP}\left(\varphi^{-1}(m)\right) \leq_{\text {mlex }} \mathrm{CP}(G) .
$$


Proof: We prove by induction on $|A|$. If $|A|=1$, the statement is trivially true. Assume $n \geq 2$ and the statement holds for $A$ with cardinality $n-1$. We will show the statement is true for $|A|=n$.

Suppose $m$ is in the form of 4.1. Let

$$
a=a_{i_{n}}, A^{\prime}=A \backslash\{a\}, m^{\prime}=\left\langle\cdots\left\langle\left\langle a_{1}, a_{i_{2}}\right\rangle, a_{i_{3}}\right\rangle, \cdots, a_{i_{n-1}}\right\rangle .
$$

Since $m$ and $G$ form a good pair. There exist $G^{\prime} \in \mathcal{R}_{A^{\prime}}^{i}$ such that $G$ is obtained from $G$ by attaching $a$ to a vertex of $G$, and $m^{\prime}$ and $G^{\prime}$ form a good pair. By induction hypothesis, we have that $\operatorname{CP}\left(\varphi^{-1}\left(m^{\prime}\right)\right) \leq_{\text {mlex }}$ $\mathrm{CP}\left(G^{\prime}\right)$.

Note that

$$
\varphi^{-1}(m)=\varphi^{-1}\left(m^{\prime}\right) \triangleleft a, \text { and } G
$$

By Lemmas 4.12 and 4.11, we have

$$
\mathrm{CP}\left(\varphi^{-1}(m)\right) \leq_{\text {mlex }} \mathrm{CP}\left(G^{\prime} \triangleleft a\right) \leq_{\text {mlex }} \mathrm{CP}(G),
$$

completing the proof.

Proof of Theorem 4.1: We define a total ordering $<$ on $\mathcal{R}_{A}^{i}$ satisfying for any $G, G^{\prime} \in \mathcal{R}_{A}^{i}$, if $\mathrm{CP}(G)<_{\operatorname{mlex}}$ $\mathrm{CP}\left(G^{\prime}\right)$, then $G<G^{\prime}$. Assume the elements in $\mathcal{R}_{A}^{i}$ is ordered as:

$$
G_{1} \prec G_{2} \prec G_{3} \prec \cdot
$$

We give an ordering on $\mathrm{Comb}_{A, 0}^{2}$ accordingly by letting $m_{i}=\varphi\left(G_{i}\right)$ :

$$
m_{1}<m_{2}<m_{3}<\cdot
$$

First, because $\varphi$ is a good-pair bijection, we clearly have that $m_{i}$ and $G_{i}$ form a good pair for each $i$.

Suppose $m_{i}$ and $G_{j}$ form a good pair. Then by Corollary 4.13 , we have

$$
\mathrm{CP}\left(G_{i}\right) \leq_{\text {mlex }} \operatorname{CP}\left(G_{j}\right)
$$

By the construction of $<$, we have $G_{i} \leq G_{j}$, which means $i \leq j$.

\section{The unique bijection between $\mathcal{R}_{A, n-1}$ and $\mathrm{Comb}_{A, n-1}^{2}$}

The main result of this section is that our conjectures are true for $i=n-1$.

Theorem 5.1 Conjecture 3.9 holds for $i=n-1$. Hence, Conjecture 1.1 holds for $i=n-1$.

The proof is very similar to what we give for the cases where $i=0$ in the last section, but with a slightly complicated construction. We omit the proof from this extended abstract. 


\section{References}

[1] M. Bershtein, V. Dotsenko, and A. Khoroshkin. Quadratic algebras related to the bi-hamiltonian operad. Int. Math. Res. Not., 2007, 2007. Article ID rnm 122, 30 pages.

[2] V. Dotsenko and A. Khoroshkin. Character formulas for the operad of two compatible brackets and for the bihamiltonian operad. Funct. Anal. Appl., 41:1-18, 2007.

[3] V. Dotsenko and A. Khoroshkin. Gröbner bases for operads. Duke Math. J., 153:363-396, 2010.

[4] B. Drake. An inversion theorem for labeled trees and some limits of areas under lattice paths. 2008. ProQuest LLC, Ann Arbor, MI. Thesis (Ph.D.)-Brandeis University.

[5] R. S. González D’león. On the free Lie algbra with multiple brackets. preprint.

[6] R. S. González D'león and M. Wachs. On the (co)homology of the poset of weighted partitions. Trans. Amer. Math. Soc. to appear.

[7] F. Liu. Combinatorial bases for multilinear parts of free algebras with two compatible brackets. J. Algebra, 323:132-166, 2009. doi:10.1016/j.jalgebra.2009.10.002.

[8] B. Vallette. Homology of generalized partition posets. J. Pure Appl. Algebra, 208:699-725, 2007.

[9] M. Wachs. On the (co)homology of the partition lattice and the free lie algebra. Discrete Math., 193:287-319, 1998. 